\title{
DELIBERACIÓN: ACTIVIDAD POLITICA EN INTERNET Y REDES SOCIALES EN COLOMBIA
}

\section{Deliberation: Political Activity on Internet and Social Networks in Colombia}

\author{
Deliberação: atividade política em internet e redes sociais na Colômbia
}

RECIBIDO: 7 DE JULIO DE 2014

Margarita M. Orozco Arbeláez (Colombia) Pontificia Universidad Javeriana de Bogotá margaraorozco@gmail.com

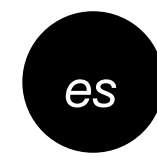

\section{RESUMEN}

En los últimos diez años una multiplicidad de estudios que han examinado el impacto positivo de los usos políticos de internet (political internet uses, PIU) y de las redes sociales en la democracia sugiere que estos son una poderosa herramienta que contribuye con la calidad de la democracia y el empoderamiento ciudadano. Sin embargo, los resultados que arrojan son heterogéneos y solo han analizado dimensiones de la democracia, como el voto, la movilización social y la participación en protestas, además de haberse concentrado en su mayoría en sociedades desarrolladas. En el presente artículo se investigan los efectos del PIU y el uso político de las redes sociales en una dimensión novedosa: la deliberación. Usando los datos de la Encuesta comunicación y participación política 2012, se analizó el impacto del PIU y de los usos políticos de las redes sociales en las actitudes deliberativas. Los resultados sugieren que el PIU y los usos políticos de las redes sociales tienen un impacto significativo y positivo en la deliberación.

PALABRAS CLAVE: Latinoamérica, Colombia, redes sociales, internet, deliberación.

EVALUADO: 15 DE SEPTIEMBRE DE 2014

ACEPTADO: 25 DE SEPTIEMBRE DE 2014

Alejandra Ortiz-Ayala (Colombia)

Magíster en Ciencia Política

Centro de Memoria Histórica Militar

alejandra.a0324@gmail.com
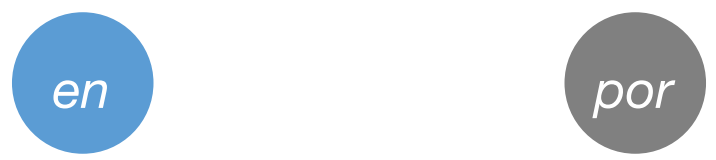

\section{ABSTRACT}

During the last ten years, several studies that have examined the positive impact political Internet (PIU) and social network uses have on democracy suggest that these are a powerful tool that contributes to its quality and the empowerment of citizens. However, the results of these studies are mixed because they have taken into account only certain aspects of democracy such as: votes, social mobilization, and participation in protests. In addition, they focus mainly on developed societies. This article researches on the effects PIU and the political use of social networks from a new perspective: deliberation. By means of the data obtained from the survey Political Communication and Participation in Colombia 2012, it was possible to analyze the impact of these two tools on deliberative attitudes. The results suggest that they have a meaningful and positive impact on deliberation.

KEYWORDS: Latin America, Colombia, social networks, Internet, deliberation.

\section{RESUMO}

Nos últimos dez anos uma multiplicidade de estudos que têm examinado o impacto positivo dos usos políticos da internet (political internet uses, PIU) e das redes sociais na democracia sugere que estes são uma poderosa ferramenta que contribui com a qualidade da democracia e o empoderamento cidadão. Porém, os resultados que arrojam são mistos e só têm analisado dimensões da democracia, como o voto, a mobilização social e a participação em protestas, além do mais de ter-se concentrado na sua maioria em sociedades desenvolvidas. No presente artigo se investigam os efeitos do PIU e o uso político das redes sociais em uma dimensão novidadeira: a deliberação. Usando os dados da enquete comunicação e participação política na Colômbia em 2012, se analisou o impacto do PIU e dos usos políticos das redes sociais nas atitudes deliberativas. Os resultados sugerem que o PIU e os usos políticos das redes sociais têm um impacto significativo e positivo na deliberação.

PalaVRAS ChaVE: Latino América, Colômbia, redes sociais, internet, deliberação.

PARA CITAR ESTE ARTÍCULO/TO CITE THIS ARTICLE/PARA CITAR ESTE ARTIGO:

Orozco Arbeláez, M. M. y Ortiz-Ayala, A. (2014). Deliberación: actividad política en internet y redes sociales en Colombia. Panorama, 8(15), 91-100. 


\section{INTERNET, REDES SOCIALES Y DEMOCRACIA}

En los últimos años, un sinnúmero de estudios ha examinado el impacto de internet en la democracia, los cuales sugieren que tiene un gran potencial en la contribución de la calidad de la democracia. La mayoría de estos estudios han mostrado que internet les ofrece a los ciudadanos un fácil acceso a la información política, al tiempo que motiva el involucramiento de la población en los asuntos de interés político (Shah et al., 2005; Tedesco, 2007; DeVreese, 2007; Wang, 2007). Internet contribuye, además, a la participación ciudadana en actividades políticas, como debates, foros, blogs y, en algunos casos, a la discusión con políticos y miembros de las instituciones (Kushin y Yamamoto, 2010; Velásquez, 2013).

Por otro lado, algunos académicos argumentan que el número de ciudadanos que usan internet con fines políticos es limitado (Rainie y Smith, 2012). De acuerdo con este punto de vista, los ciudadanos que usan internet con fines políticos son aquellos que están naturalmente predispuestos o interesados en política y para los cuales internet es apenas una herramienta de participación más (Boulianne, 2009; Kruikemeier et al., 2013).

Esto demuestra que la investigación ha reportado resultados contradictorios, con lo cual no es posible establecer con total certeza que internet motiva el involucramiento político y, quizás, estemos sobreestimando su impacto (Bimber, 2003). De hecho, quienes usan internet con fines políticos son ciudadanos más sofisticados políticamente, quienes tienen más ventajas al usarlo como fuente de participación en la vida pública, lo cual muestra una importante brecha en los beneficios que se le han venido atribuyendo en la democracia. En resumen, la evidencia no es consistente sobre su potencial papel en las democracias contemporáneas.

Los resultados mixtos que han sido hallados en pasados estudios están relacionados con ciertas formas de participación, tales como el voto, la movilización y la participación en protestas. Sin embargo, estos estudios no consideraron un aspecto clave de la participación política: la deliberación. Internet puede ser un espacio ideal para generar deliberación política porque permite la libre expresión de pensamientos e ideas y las personas pueden exponerse a una multiplicidad de puntos de sociales, puede promover el diálogo y la interacción entre los ciudadanos operando como un mecanismo que facilita el mutuo entendimiento entre los miembros de una comunidad, así, los medios pueden ayudar a supervisar y comprender el contexto, proveer información a las personas y hacerlas más conscientes de las oportunidades que ellos tienen cuando participan en política (Orozco y Ugarriza, 2014). Las redes sociales también tienen el potencial de fortalecer ciertas motivaciones que facilitan algunos niveles de participación cívica, lo cual permite que las personas trabajen de manera conjunta en la resolución de problemas comunes.

El presente estudio entiende la deliberación desde la perspectiva de Habermas (1998), según la cual esta es una expresión de compromiso compartido que busca el interés común. Se trata de un acto en el que los ciudadanos consideran hechos relevantes desde diferentes puntos de vista y dialogan con los demás para pensar críticamente sobre las distintas opciones que tiene un asunto público; así, se incrementan las perspectivas, compresiones y opiniones sobre un tema específico.

Recientemente, algunos académicos han tratado de medir los niveles de deliberación entre excombatientes colombianos. De esta forma, investigadores de la Universidad de Berna invitaron a un grupo de esta población a hablar de política como una forma de determinar las ventajas y desventajas del modelo deliberativo en contextos de violencia. Así, por medio de un diseño cuasiexperimental, se analizó el desempeño deliberativo de los exguerrilleros y exparamilitares en grupos de discusión. Los resultados mostraron que, en condiciones adecuadas, se puede esperar un comportamiento altamente deliberativo, incluso en contextos de profunda hostilidad y serios desacuerdos. El estudio identificó una serie de factores que incidieron positivamente en los niveles de deliberación, como un conjunto particular de reglas para la discusión (por ejemplo el consenso), un número pequeño de participantes, disposiciones psicológicas más democráticas y actitudes más pluralistas (Ugarriza, 2012).

Consideramos que la deliberación es un aspecto muy importante para la democracia, porque unas instituciones y una ciudadanía más deliberativas se pueden reforzar mutuamente y reducir el impacto de ciertos factores de violencia. De esta manera, Colombia ofrece una única oportunidad para dar un giro interesante a 
las preguntas por el mejoramiento de la calidad de la democracia y promover el modelo deliberativo (Orozco y Ugarriza, 2014).

Con el ánimo de explorar el potencial de la deliberación, la Encuesta comunicación y participación politica 2012, realizada por el Centro de Investigación en Comunicación Política de la Universidad Externado de Colombia, incluyó una serie de preguntas que intentaban medir la percepción deliberativa de los ciudadanos siguiendo las seis características de la deliberación: participación, respeto, calidad de los argumentos, evaluación del bien común, capacidad de ceder ante los mejores argumentos y sinceridad (Dryzek, 2009).

Con respecto a al uso político de internet (political internet use [PIU]), los estudios que han reportado diferentes hallazgos en el tema se han llevado a cabo en países con democracias maduras. Así, resulta importante explorar cuáles son las dinámicas de uso político de internet en países con democracias menos robustas y de menos acceso tecnológico, como Latinoamérica y países de Medio Oriente. Por democracias desarrolladas entendemos países que encarnan los valores de libertad e igualdad en torno a una institución política fuerte. De esta manera, la calidad de la democracia está relacionada con el respeto de los derechos civiles básicos. En contraste, las democracias no desarrolladas tienen instituciones débiles y dificultades para proteger los derechos civiles básicos de los ciudadanos.

Un estudio reciente que examina el impacto del PIU en la democracia en varios países de Medio Oriente encontró que el contexto político desempeña un papel muy importante en los efectos del PIU en la democracia. La investigación demuestra que los sistemas políticos represivos condicionan el impacto positivo que puede tener internet en la política. En este tipo de contextos, las redes sociales reducen las barreras para llevar a cabo acciones colectivas e incentivan la movilización política. De esta manera, las personas estarían más dispuestas a participar en política a través de las redes en regímenes más autoritarios que en los democráticos (Bridwell, 2013).

Los estudios más recientes sobre los efectos del PIU en la participación política han encontrado resultados disímiles entre los usos pasivos y activos del PIU. Los usos activos se definen como una participación bidimensional

que facilita la interacción en contraste con los usos pasivos que solo incluyen la comunicación unidireccional. Los resultados sugieren que los usos activos tienen un mayor efecto en la participación política y en el involucramiento político (Dimitrova et al., 2011; Kruikemeier et al., 2013; Zúñiga, Puig-I-Abril y Rojas, 2009). Otro factor que podría estar mediando entre los efectos del PIU y la participación política está relacionado con las plataformas disponibles para los usos activos o pasivos del PIU.

Esta es otra evidencia que sugiere que el impacto positivo de internet depende de las condiciones sociodemográficas de las personas que deciden hacer uso del PIU en diferentes países. Por ejemplo, el estudio LAPOP (The Latin American Public Opinion Project) muestra que las personas más jóvenes, más adineradas y más educadas que viven en las áreas urbanas son las que más participan en política a través de internet y las redes sociales en América Latina (Brunelle, 2013).

De esta manera, Colombia es uno de los casos en los cuales resulta interesante estudiar los efectos positivos del PIU, ya que este país, a pesar de tener la democracia más antigua de América Latina, presenta también uno de los conflictos armados más antiguos del mundo.

Como ya se había mencionado, unas instituciones y una ciudadanía más deliberativas pueden reducir el impacto de ciertos factores de violencia y contribuir, por tanto, a canalizar el conflicto propio de las sociedades.

Carlo Nasi evidenció cómo ciertos grados de violencia política implican la violación de ciertas precondiciones necesarias para establecer un Estado como democrático, tales como la libertad de expresión, la fuentes alternativas de información y la libre organización, y sugiere que,

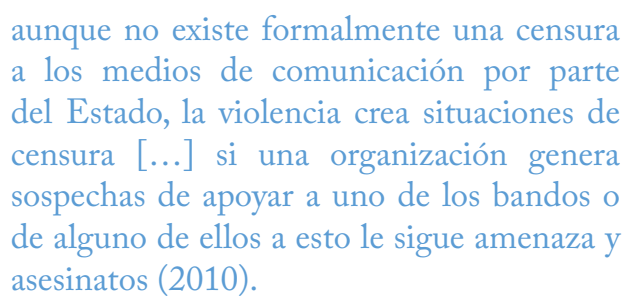

En Colombia, el conflicto armado interno que ha estado por más de sesenta años en el país ha hecho que las estructuras institucionales y lógicas de la cultura democrática adquieran una naturaleza de polarización 
ideológica de tal magnitud que cualquiera que se oponga al statu quo es catalogado como "guerrillero". Es común que aquellos que tengan una ideología de izquierda se asocien directamente con agrupaciones insurgentes.

Margarita

M. Orozco

Arbeláez I

Alejandra

Panorama I

Algunos académicos son pesimistas acerca de la habilidad de los medios para crear verdaderos espacios para la deliberación (la televisión e internet tienen la peor repu-

Frente a los efectos de una guerra prolongada

[se puede plantear] que en Colombia se ha generado una especie de "normalización" de la relaciones deshumanizantes [...] la violencia [es] un acto comunicativo (pues "nos dice algo") que paradójicamente es expresión de los límites de "aceptación del otro junto a uno", es decir que pone en evidencia, al mismo tiempo, la capacidad de aceptar la diferencia como parte de la interrelación humana y puede ser, al mismo tiempo, el obstáculo o la negación de esta aceptación (lo heterogéneo o lo diferente) que facilita asumir las banderas a favor del aniquilamiento del otro y/o su invisibilización como sujeto (Centro Nacional de Memoria Histórica, 2014, pp. 89-91).

De ahí que sea importante estudiar la deliberación en Colombia, pues la democracia en sentido estricto no se caracteriza por el conflicto, sino por el disenso, ya que como afirma Sartori (1976): "la diferencia y no la semejanza, la discrepancia y no la unanimidad, el cambio y no la inmutabilidad constituyen el buen vivir". Lo cual ha sido bastante difícil de desarrollar en la democracia colombiana. Adicionalmente, Colombia es también uno de los países más inequitativos de la región (Portafolio. co, 2013).

Un estudio sobre los usos políticos de internet en Colombia muestra que los ciudadanos que usan las redes sociales con fines políticos son más proclives a participar en protestas sociales y mucho menos en las elecciones políticas (Ortiz y Merchán, 2013). Así, el efecto positivo del PIU en Colombia ha sido examinado solo en dos dimensiones de la participación política (movilización y voto), pero el conocimiento es bastante escaso en aspectos importantes para la democracia, como la deliberación.

\section{DELIBERACIÓN, CONVERSACIÓN Y MEDIOS} tación), en cuanto lo que se favorece es la privatización, los ratos libres, y las personas prefieren gastar el tiempo en entretenimiento en lugar de llevar a cabo actividades cívicas (Putnam, 2000). A la televisión también se le culpa de tener un efecto nocivo sobre la confianza entre las personas, ya que presenta un mundo violento y hostil (Gerbner et al., 1980). El mismo Habermas (1996, 1998) menciona la dificultad que presentan los medios en la constitución de una verdadera esfera pública, ya que estos están al servicio del poder, promoviendo una conformidad social y unos patrones homogéneos de consumo.

Sin embargo, el modelo de mediación comunicativa ofrece evidencia de que el uso de medios en conjunto con la conversación interpersonal sobre temas políticos genera más integración social y participación cívica (McLeod et al., 1996). Las actividades comunicacionales no solo tienen un efecto directo en el comportamiento participativo, sino también un impacto indirecto, ya que se incrementa el conocimiento político que trae consigo una mayor participación (McLeod et al., 1999).

Con respecto al uso de las nuevas tecnologías, diferentes estudios han encontrado una relación positiva entre los usos informativos de internet y los niveles de capital social (Shah, Kwak y Holdbert, 2001), participación política (Shah et al., 2002) y participación cívica (Jennings y Zeitner, 2003). Otros académicos como Wellman et al. (2001) han mostrado cómo las interacciones comunicacionales en internet complementan las personales y promueven la participación.

Hasta el momento los estudios del efecto del PIU en la democracia se han concentrado en la participación política a través del voto, la movilización y la protesta social. Sin embargo, la teoría democrática deliberativa invita a pensar sobre la calidad del comportamiento político partiendo de la idea de que los espacios de interlocución política en condiciones de libre participación, respeto e intercambio de argumentos pueden fomentar la legitimidad y validez de la toma de decisiones por ser productos del consenso (Cohen, 1989; Habermas, 1996; Steiner, 2012).

De ahí que la propuesta de este artículo sea evaluar los efectos del PIU en la liberación política, pues consideramos que el espacio de internet y las redes sociales puede fomentar la capacidad de disposición de los ciudadanos para ceder ante los mejores argumentos, ya que están 
expuestos a diferentes puntos de vista; más concretamente se espera que el PIU afecte la deliberación ciudadana de forma significativa y positiva en aquellos que la usan frente a aquellos que no. Se espera también encontrar la misma relación en lo referente al uso de las redes sociales con fines políticos. De esta manera, las hipótesis de este trabajo son las siguientes:

Hipótesis 1A: el PIU tiene un impacto significativo y positivo en la deliberación frente a aquellos que no usan internet con fines políticos.

Hipótesis 1B: el uso de redes sociales con fines políticos tiene un impacto significativo y positivo en la deliberación frente a aquellos que no usan redes sociales con fines políticos.

Incluiremos variables de conversación política para controlar nuestro modelo, así se espera que las personas que hablan de política con su familia y amigos tengan mayores niveles de deliberación. Al igual que aquellos que hablan con personas que tienen ideas diferentes de las propias.

Hipótesis 2A: los ciudadanos que hablan con sus familias sobre política o sobre las noticias tienen mayores niveles de deliberación.

Hipótesis 2B: los ciudadanos que hablan con sus familias y amigos sobre política o sobre las noticias tienen mayores niveles de deliberación.

Hipótesis 2C: los ciudadanos que hablan con personas con distintas ideas de las propias tienen mayores niveles de deliberación.

Este artículo usa los datos de la Encuesta comunicación y participación politica en Colombia $2012^{1}$. Esta es la más completa encuesta sobre consumo y uso de medios y participación política en Colombia. Se usaron los datos recolectados sobre los diferentes usos políticos de internet y las redes sociales.

Finalmente, este estudio pretende contribuir, con un indicador proxy, a los usos políticos de internet y las redes sociales. También se pretende entregar un indicador proxy sobre la deliberación política. Es la primera vez que intenta medirse de manera empírica la percepción deliberativa en Colombia a través de una encuesta; por eso, este estudio representa un esfuerzo por explorar un fenómeno que no ha sido estudiado antes de esta manera en la región.

\section{DATOS Y MÉTODO}

Para examinar los efectos del PIU y las redes sociales con fines políticos, se usó la encuesta nacional de una muestra representativa de la población urbana en Colombia. Específicamente, se usó un modelo de regresión lineal múltiple de mínimos cuadrados ordinarios.

Para validar las hipótesis, se especificó un modelo para la variable dependiente sobre el índice de deliberación, que fue construido tomando como base las seis características de la deliberación establecidas por Dryzek (2009): participación, respeto, calidad de los argumentos, evaluación del bien común, capacidad de ceder ante los mejores argumentos y sinceridad. Esta estuvo basada en las siguientes respuestas de los ciudadanos:

D45. Usando una escala de 0 a 5 donde 0 significa completamente en desacuerdo y 5 completamente de acuerdo. ¿Qué tanto usted está de acuerdo o en desacuerdo con las siguientes afirmaciones? Cuándo yo hablo de política con otra gente generalmente:

D45A. Tengo amplia oportunidad de expresar mis ideas.

D45C. Creo que se lograron buenos argumentos en la discusión.

D45E. Trato siempre de apelar al bien común.

D45G. Trato de expresar las ideas que de verdad tengo en mente.

D45I. Trato a todo el mundo con respeto durante la discusión.

D45L. Tiendo a ceder ante mejores argumentos.

\footnotetext{
y las redes sociales. También se pretende entregar un
}

I Panorama I pp. 91-100 I Volumen 8 I Número 15 I Julio-diciembre | 2014 
Se recodificaron y promediaron las respuestas a estas preguntas, las cuales fueron originalmente hechas de 0 a 5 y se creó el índice Deliberation, que va de 0 (completamente en desacuerdo) a 100 (completamente de acuerdo) $)^{2}$.

M. Orozco

Arbeláez I

Alejandra

En orden de testear las hipótesis, se necesitó una medida individual para cada respuesta sobre el PIU. De esta manera, se usó un proxy que midiera las respuestas de los participantes sobre las preguntas que se describen a continuación:

C15: en una escala de 0 a 5 donde 0 significa nunca y 5 frecuentemente. ¿Qué tan frecuentemente usted realiza las siguientes actividades en internet? ${ }^{3}$

C15B: usar internet para discutir noticias de actualidad y temas de política.

C15H: visitar blogs políticos.

C15K: buscar información gubernamental.

C15N: hacer contribuciones a una campaña.

C15O: vincularse como voluntario de una causa o partido político.

C15P: apoyar protestas políticas.

Nuestro argumento está centrado en el hecho de que los ciudadanos que usan internet para participar políticamente son más deliberativos porque encuentran en este un ambiente con diversidad de puntos de vista, que los expone a un mundo más heterogéneo. Así, las personas que se exponen a esta variable tienden a ser más deliberativos que aquellos que no usan internet con este propósito.

Por otro lado, incluimos dos preguntas diferentes para capturar otro tipo de usos políticos de internet, particularmente, las redes sociales. Esto se hizo de acuerdo con lo que las personas reportaron sobre las siguientes preguntas:

C21. ¿Cómo usa las redes sociales? En una escala de 0 a 5 donde 0 significa nunca y 5 frecuentemente. ¿Qué tan frecuentemente usted realiza las siguientes actividades en sitios de redes sociales? ${ }^{4}$

C21D: compartir historias periodísticas con los contactos.

C21E: movilizar los contactos alrededor de causas sociales o políticas.

Como lo sugiere nuestra hipótesis, el uso de redes sociales con fines políticos afecta positivamente los niveles de deliberación de los ciudadanos.

La tercera pregunta tiene relación con la actividad política en las redes sociales que muestra evidencia de cuándo las personas tienen bajos niveles de deliberación. El fenómeno se denomina Hide Comment, y hace referencia a que una persona oculta un comentario porque no comparte su punto de vista político. Con una variable dicotómica codificada 1 , si el encuestado respondió sío no (y 0), la pregunta es la siguiente:

C23. ¿Alguna vez ocultó los comentarios de alguien de su círculo porque no comparte sus opiniones políticas?5

Nuestro argumento teórico no se limita al impacto del PIU y el uso político de las redes sociales. Queremos contrastar el impacto de las nuevas herramientas de la información política y los nuevos espacios de comunicación política con los espacios tradicionales de conversación política sin el uso de internet. Esta variable está incluida en el análisis para controlar factores que la literatura ha relacionado con la deliberación.

Primero, incluimos en el análisis una medida de las respuestas sobre traditional political conversation con dimensiones compuestas por dos aspectos:

\footnotetext{
4 Se recodificaron las preguntas a una escala de 0-100, donde 0 significa nunca y 100 frecuentemente.

5 Se recodificaron estas preguntas en una escala de 0-100 donde 0 significa no y 100 si.
}

2 Este índice es confiable. The Cronbach's alpha es 0.80 en 2012, para las observaciones incluidas en el modelo.

3 Se recodificaron estas preguntas en una escala de $0-100$, donde 0 significa nunca y 100 frecuentemente. Este índice es confiable. The Cronbach's alpha es 0.847 para la observación incluida en el modelo. 
1) ¿Qué tan frecuentemente usted comenta las noticias o habla de política con?

D42A: miembros de la familia.

D42D. Colegas o amigos.

2) Pensando en las personas con las cuales usted comenta las noticias o habla de política, en una escala de 0 a 5 , donde 0 es nunca y 5 frecuentemente, ¿qué tan frecuentemente usted habla con personas que...? ${ }^{7}$

D42A: tienen diferentes ideas a las suyas.

También se incluyeron algunos controles sociodemográficos, como sexo (femenino codificado con 1 y masculino con 0 ), nivel de educación medido por número de años completados, edad en años cumplidos y nivel socioeconómico calculado en ingresos económicos mensuales.

\section{RESULTADOS}

\section{EL PIU Y LAS REDES SOCLALES Y SU IMPACTO EN LA} DELIBERACIÓN

El modelo 1 de la tabla 1 es un modelo de base que incluye todas las variables independientes que se describen en la sección anterior. Los resultados se apoyan en la hipótesis sobre el efecto positivo del PIU en la deliberación ciudadana. De hecho, entre más actividad política en internet y más usos políticos de las redes sociales que hacen los encuestados, más deliberativos son. Sin embargo, al contrario de nuestras expectativas, ocultar comentarios de los otros en las redes sociales por razones políticas no tiene un impacto significativo en la deliberación.

\section{ESPACIOS TRADICIONALES DE CONVERSACIÓN POLÍTICA Y DELIBERACIÓN}

Factores determinantes incluidos en el modelo relacionado con la conversación política tradicional revelan que

\footnotetext{
6 Se recodificaron estas preguntas en una escala de 0-100 donde 0 significa nunca y 100 frecuentemente.

7 Se recodificaron estas preguntas en una escala de 0-100 donde 0 significa nunca y 100 frecuentemente.
}

los ciudadanos que hablan con frecuencia con miembros de la familia acerca de las noticias o de política son más deliberativos.

Por otro lado, los encuestados que dijeron hablar frecuentemente con su familia y amigos acerca de las noticias o de la política fueron en la dirección opuesta de nuestras hipótesis. Sin embargo, estos resultados no son estadísticamente significativos. Por último, los ciudadanos que hablan con personas que tienen ideas muy diferentes de las suyas son más deliberativas. Este resultado confirma nuestra hipótesis sobre el impacto positivo de la conversación con personas que tienen ideas diferentes sobre los niveles de deliberación.

IMPACTO DE LAS VARLABLES SOCIODEMOGRÁFICAS EN LA DELIBERACIÓN

El nivel de educación tiene un efecto positivo y significativo en la deliberación. Al contrario de nuestras expectativas, las personas que tienen más dinero son menos deliberativas.

\section{DISCUSIÓN}

1) Los resultados de este estudio mostraron que las personas más adineradas tienden a ser menos deliberativas. Estos hallazgos podrían estar relacionados con el contexto de desigualdad que caracteriza a Colombia. De acuerdo con datos de las Naciones Unidas, Colombia es el tercer país más desigual de América Latina. En un contexto de desigualdad, es difícil que la gente tome acciones racionales que incluyan beneficios colectivos, ya que el ejercicio de la deliberación implica costos y tiempo que no todos están dispuestos a asumir. Especialmente, las personas saben que resolver el problema por sí mismos es la manera más rápida y eficiente de llegar a una solución, en lugar de discutir con otros acerca de un tema en particular.

2) Como la hipótesis lo sugiere, los usos políticos de internet y redes sociales tienen un impacto positivo en la deliberación política. Por lo tanto, es posible pensar que internet y las redes sociales son espacios ideales para que los ciudadanos desarrollen habilidades, como el respeto, la tolerancia, la interacción con diversos puntos de vista y mejoren sus cualidades argumentativas. Por otro lado, es importante leer estos resultados a la luz

I Panorama I pp. $91-100$ I Volumen 8 I Número 15 | Julio-diciembre 12014 
de otros hallazgos que han sugerido que las personas que utilizan internet para las actividades políticas son más tolerantes, pero también políticamente más polarizadas. De acuerdo con estos resultados, pensamos que los colombianos que utilizan internet con fines políticos y las redes sociales están expuestos a diversas opiniones que les permitan desarrollar una capacidad para aceptar y convivir con diferentes puntos de vista distintos de los propios. Lo interesante aquí es que la exposición no garantiza que estén más dispuestos a ceder ante los argumentos de los demás. En realidad, lo que ocurre es que las predisposiciones se refuerzan. Es decir, gracias a la exposición a los diferentes puntos de vista, los colombianos podrían construir mejores argumentos a favor de sus predisposiciones solo por estar expuestos a ideas contrarias, lo cual, en última instancia, garantiza un ambiente más amigable para el debate público, pero, quizá, más polarizado.

3) Algunos estudios señalaron que la inhibición de la expresión se desarrolla tempranamente. De hecho, el factor más importante de predisposición de las personas a una conversación sobre temas políticos no es la educación, sino la experiencia de la comunicación en la familia y la escuela (Rojas y Gil de Zúñiga, 2010; Rojas y Hopke, s. f.). Estos resultados destacan la importancia de promover algunas prácticas deliberativas en el hogar, con un ambiente más favorable a la expresión y al intercambio de ideas. Nuestros resultados coincidieron con los hallazgos citados antes y sugieren la importancia de la conversación y la exposición a diferentes ideas y en diferentes lugares durante la infancia para construir una cultura más deliberativa y tolerante.

4) Para terminar, queremos poner énfasis en un resultado muy interesante para el caso colombiano. Después de más de cincuenta años de conflicto armado interno, el país está llevando a cabo un proceso de paz, lo cual ha producido una fuerte polarización política entre la población. La formación de la opinión pública en torno a este tema está hoy fuertemente influenciada por la información que circula en internet y las redes sociales. Los resultados que aparecen en este estudio son alentadores, ya que muestran cómo los ciudadanos comunes y corrientes que hacen usos políticos de internet y de las redes sociales pueden desarrollar mejores habilidades para la deliberación y la tolerancia política.

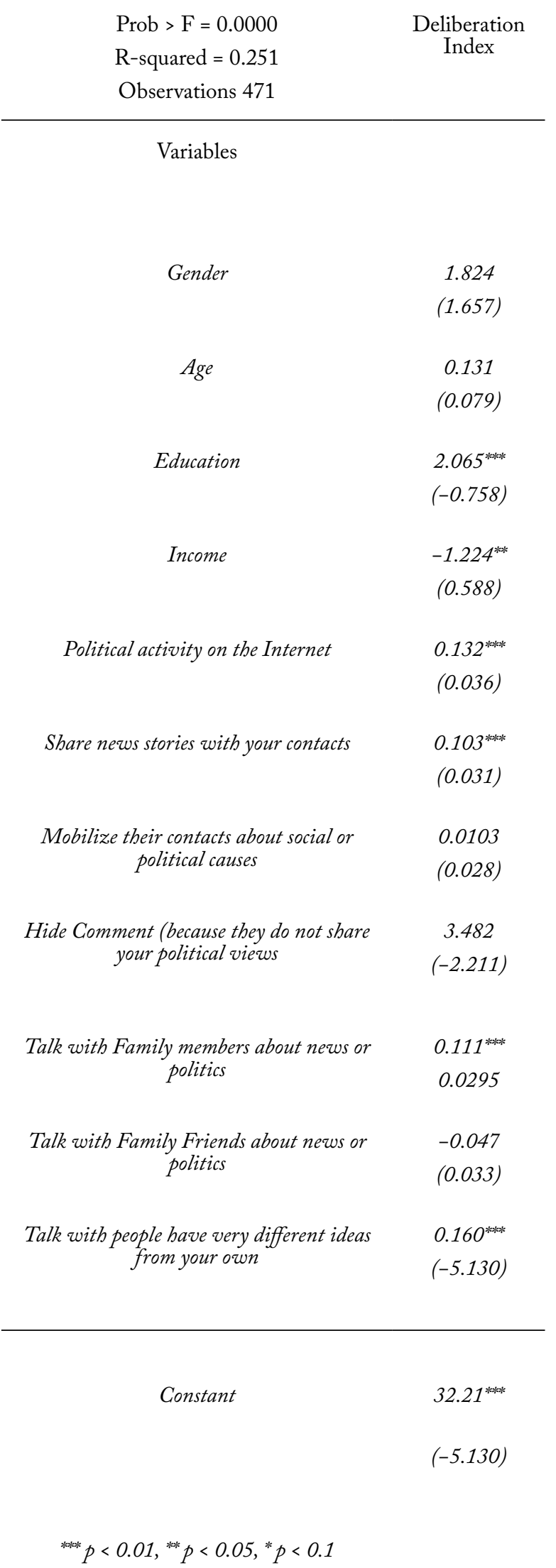

Tabla 1. Explaining the effects of PIU and social network political use on Deliberation Index 


\section{REFERENCIAS BIBLIOGRÁFICAS}

1. Bimber, B. A. (2003). Information and american democracy: technology in the evolution of political power. Cambridge, UK, Nueva York: Cambridge University Press.

2. Boulianne, S. (2009). Does internet use affect engagement? A meta-analysis of research. Political Communication, 26(2), 193-211.

3. Bridwell, J. M. H. (2013). Twitter, texting, and street demonstrations: assessing social media's political relevance for citizen empowerment. En APSA 2013 Annual Meeting Paper.

4. Brunelle, J. (2013). Political social media users i $\mathrm{n}$ the americas are tolerant and pro-democratic, Number, 92, 1-7.

5. Centro Nacional de Memoria Histórica (2014). Desaparición forzada tomo III: entre la incertidumbre y el dolor: impactos psicosociales de la desaparición forzada. Bogotá: Centro Nacional de Memoria Histórica.

6. Cohen, J. (1989). Deliberation and democratic legitimacy. En A. Hamlin y P. Pettit (eds.), The good polity. Normative analysis of the state (pp. 17-34). Oxford: Basil Blackwell.

7. De Vreese, C. H. (2007). A spiral of euroscepticism: the media's fault? Acta Política, 42(2), 271-286.

8. Dimitrova, D. V., Shehata, A., Strömbäck, J. y Nord, L. W. (2011). The effects of digital media on political knowledge and participation in election campaigns: evidence from panel data. Communication Research, 0093650211426004.

9. Dryzek, J. S. (2009). Democratization as deliberative capacity building. Comparative Political Studies, 42(11), 1379-402.

10. Gerbner, G., Gross, L., Morgan, M. y Signorielli, N. (1980). The "mainstreaming" of America: violence profile n.o 11 . Journal of Communication, 30(3), 10-29.

11. Habermas, J. (1996). Between facts and norms. Contributions to a theory of law and democracy (trad.William Rehg). Cambridge: Cambridge University Press.

12. Habermas, J. (1998). The inclusion of the other. Cambridge, MA: MIT Press.

13. Kushin, M. J. y Kitchener, K. (2009). Getting political on social network sites: exploring online political discourse on Facebook. First Monday, 14(11). Recuperado de http:// firstmonday.org/ojs/index.php/fm/article/ view/2645
14. Kushin, M.J.y Yamamoto, M. (2010). Did social media really matter? College students' use of online media and political decision making in the 2008 election. Mass Communication and Society, 13(5), 608-630.

15. Kruikemeier, S., Noort, G. van, Vliegenthart, R. $y$ Vreese, C. H. de (2013). Getting closer: the effects of personalized and interactive online political communication. European Journal of Communication, 0267323112464837.

16. McLeod,J. M., Daily, K., Guo, Z., Eveland, W. P. Jr., Bayer, J., Yang, S. et al. (1996). Community integration, local media use, and democratic processes. Communication Research, 23, 463-487.

17. McLeod,J. M., Zubric, J., Keum, H., Deshpande, S., Cho, J., Stein, S. y Heather, M. (2001, agosto). Reflecting and connecting: testing a communication mediation model of civic participation. En Annual meeting of the Association for Education in Journalism and Mass Communication Washington, DC.

18. McLeod, J. M., Scheufele, D., Moy, P., Horowitz, E. M., Holbert, R. L., Zhang,W., Zubric, S. y Zubric, J. (1999). Understanding deliberation: the effects of discussion networks on participation in a public forum. Communication Research, 26, 743-774.

19. Nasi, C. (2010). Guerras de guerrillas, acuerdos de paz y regímenes políticos: el problema de las clasificaciones. En A. Rettberg (coord.), Conflicto armado, seguridad y construcción de paz en Colombia (pp. 69-96). Bogotá: Universidad de los Andes.

20. Ortiz-Ayala, A. y Merchán Rincón, L.F. (2013). Internet: un nuevo instrumento de participación política. Apuntes de cultura política desde el Observatorio de la Democracia.

21. Orozco, M. y Ugarriza, J. E. (2014). The citizens, the politicians and the cohorts: a preeliminary assesment of deliberative capacity in Colombia. En D. Caluwaerts y J. E. Ugarriza, Deliberation against all odds: the potencial and pittfalls of talking politics in deeply divided societies. Londres: Palgrave McMillan.

22. Portafolio.co (2013, 18 de abril). Colombia, fuera de los 10 países más desiguales. Recuperado de http://www.portafolio.co/economia/ colombia-fuera-los-10-paises-mas-desiguales

23. Putnam, R. D. (2000). Bowling alone: the collapse and revival of american community. Nueva York: Simon \& Schuster.

24. Rainie, L.y Smith, A. (2012). Politics on social networking sites. Washington, DC: Pew Research Center. Recuperado de http://www. pewinternet.org/ /media/Files/Reports/2012/ PIP_PoliticalLifeonSocialNetworkingSites.pdf 
25. Rojas, H. y Hopke, J. (s. f.). Socializados para la autocensura: comunicación autoritaria y opinión.

26. Rojas, H. y Gil de Zúñiga, H. (2010). Comunicación y participación política en Colombia. En H. Rojas, I. Pérez y H. Gil de Zúñiga, Comunicación y comunidad (pp. 13-67). Bogotá: Universidad Externado de Colombia.

Alejandra

27. Rojas, H. y Puig-I-Abril, E. (2009). Mobilizers mobilized: information, expression, mobilization and participation in the digital age. Journal of Computer Mediated Communication, 14, 902-927.

28. Shah, V., Nojin Kwak, R. Lance Holbert, D. (2001). "Connecting" and "disconnecting" with civic life: patterns of Internet use and the production of social capital. Political Communication, 18(2), 141-162.

29. Shah, D. V., Watts, M. D., Domke, D., y Fan, D. P. (2002). News framing and cueing of issue regimes: explaining Clinton's public approval in spite of scandal. Public Opinion Quarterly, 66(3), 339-370.

30. Shah, D. V., Cho, J., Eveland, W. P., y Kwak, N. (2005). Information and expression in a digital age modeling Internet effects on civic participation. Communication research, 32(5), 531-565.

31. Steiner, J. (2012). The foundations of deliberative democracy: empirical research and normative implications. Cambridge: Cambridge University Press.

32. Tedesco, J. C. (2007). Examining internet interactivity effects on young adult political information efficacy. American Behavioral Scientist, 50(9), 118-1194.

33. Ugarriza,J. E. (2012). Potential for deliberation among ex combatants in Colombia (Tesis doctoral, University Library).

34. Velásquez, A., y Lampe, C. (2013). El papel de las valoraciones como señales en la participación en los sitios web de noticias políticas generados por el usuario. Palabra Clave, 16(3), 699-728.

35. Vreese, C. H. de (2007). Digital renaissance: young consumer and citizen? The ANNALS of the American Academy of Political and Social Science, 611(1), 207-216.

Volumen 8

Número 15

37. Wellman, B., Haase, A. Q. Witte, J., y Hampton, K. (2001). Does the Internet increase, decrease, or supplement social capital? Social networks, participation, and community commitment. American behavioral scientist,45(3) 436-455.

36. Wang, S.-I. (2007). Political use of the internet, political attitudes and political participation. Asian Journal of Communication, 17(4), 381-395.
38. Zúñiga, H. G. D., Puig-I-Abril, E. y Rojas, H. (2009). Weblogs, traditional sources online and political participation: an assessment of how the internet is changing the political environment. New Media E' Society, 11(4), 553-574. 